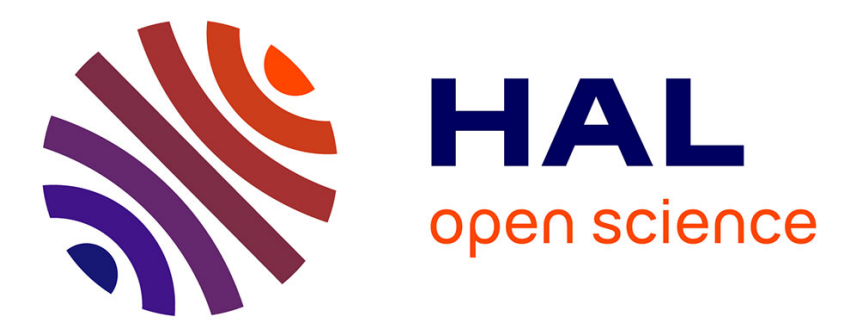

\title{
Tevatron Wij anomaly for a model with two different mechanisms for mass generation of gauge fields
}

Elias Koorambas

\section{To cite this version:}

Elias Koorambas. Tevatron Wij anomaly for a model with two different mechanisms for mass generation of gauge fields. Int J Theor Phys, 2012, 51 (10), pp.3127-3140. 10.1007/s10773-012-1194-7 . hal-00627552

\section{HAL Id: hal-00627552 \\ https://hal.science/hal-00627552}

Submitted on 29 Sep 2011

HAL is a multi-disciplinary open access archive for the deposit and dissemination of scientific research documents, whether they are published or not. The documents may come from teaching and research institutions in France or abroad, or from public or private research centers.
L'archive ouverte pluridisciplinaire HAL, est destinée au dépôt et à la diffusion de documents scientifiques de niveau recherche, publiés ou non, émanant des établissements d'enseignement et de recherche français ou étrangers, des laboratoires publics ou privés. 


\title{
Tevatron $W i j$ anomaly for a model with two different mechanisms for mass generation of gauge fields
}

\author{
E.Koorambas \\ 8A Chatzikosta, 11521 Ampelokipi, Athens, Greece \\ E-mail:elias.koor@gmail.com
}

(September 29, 2011)

\begin{abstract}
The latest Collider Detector at Fermilab (CDF) anomaly, the excess of dijet events in the invariant-mass window $120-160 \mathrm{GeV}$ in associated production with a $\mathrm{W}$ boson, can be explained by a new neutral vector $C$-boson of mass $(145 \mathrm{GeV})$ that is predicted by the $\mathrm{Wu}$ mechanisms for mass generation of gauge field. The Standard Model (SM) W, Z-bosons normally get its masses through the coupling with the SM Higgs of mass $114-200 \mathrm{GeV}$. Here, the C boson has negligible couplings to leptons, and so is not affected by the dilepton $\mathrm{C}$ constraints.
\end{abstract}

PACS Numbers: 12.15-y, 11.15-q, 11.10-z, 12.10-g

Keywords: Collider Detector Fermilab, electroweak, interactions, gauge field, symmetry breaking

\section{Contents}

1. Introduction............................................................ 2

2. The Lagrangian of the model ......................................... 2

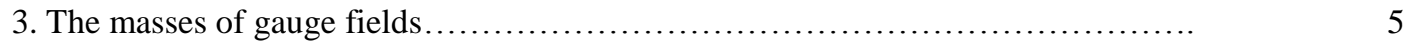

4. The Phenomenology of the mode........................................ 9

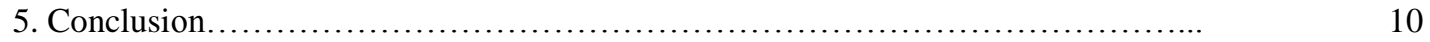

References............................................................. 10

This work is licensed under the Creative Commons Attribution 3.0 Unported License. To view a copy of this license, visit http://creativecommons.org/licenses/by/3.0/ or send a letter to Creative Commons, 444 Castro Street, Suite 900, Mountain View, California, 94041, USA. 


\section{Introduction}

The latest surprise presented by the Fermilab's Collider Detector (CDF) experiment, is an excess in the invariant-mass window of $120-160 \mathrm{GeV}$ in the dijet system of the associated production of a $W$ - boson with 2 jets [1], [2], [3],[4]. We shall denote it by Wjj production.

The hypothesis behind Fermilab's CDF experiment [1], [2], [3], [4] predicted that the number of the events - producing a $\mathrm{W}$ boson and a pair of jets - would fall off as the mass of the jet pair increased. The CDF experimental data, however, showed something strange (see [2]): a bump in the number of events when the mass of the jet pair was about $145 \mathrm{GeV}$. The excess of events in the window $\mathrm{M}_{\mathrm{ii}} \sim 120-160 \mathrm{GeV}$ appears to be a resonance. It is reportedly at 4.8 sigma, tantalizingly close to five-sigma certainty [2], [3].

However, DZero (D0) [27], have cross-checked the observation with their own independent data and analysis tools, and have found no evidence of a new particle. The detectors are somewhat different in design and in the selection criteria used to analyze events. It is certainly possible for CDF to see something that DZero has missed.

From the distribution of the events in the CDF experiment we can see that the width of the resonance appears to be slightly wider than the SM Z- boson.

The candidate particle may not belong to the standard model of particle physics. Instead, as some argue [5], [6], [7], it might be the first hint of a new force of nature, termed technicolour. This force would resolve some problems such as the "naturalness problem" [28] with the standard model, but predicts the existence of many light particles that should have been detected by now [28].

Most workers agree that the mysterious particle produced by the CDF experiment is not the long-sought Higgs boson [8, 9, 10, 11, 12, 13, 14, 15], believed by many to endow particles with mass. If it were, the bump in the experimental data would be 300 times smaller. What's more, a Higgs particle would probably decay into bottom quarks, which seem to be absent from the Fermilab data [1], [2], [3], [4].

In this paper, we investigate the nature of this mysterious particle and propose a model with two different mechanisms for mass generation of gauge fields. The Tevatron Wij anomaly is explained by a new neutral vector $C$-boson of mass $(145 \mathrm{GeV})$, as predicted by the $\mathrm{Wu}$ mechanisms for mass generation of gauge fields [16], [17], [18], [19]. The SM $W, Z$-bosons normally acquire their masses through their coupling with the SM Higgs boson, mass 114-200 $\mathrm{GeV}$ [20], [21], [22], [23]. The $C$ boson has negligible couplings to leptons, and so is not affected by the dilepton $\mathrm{C}$ constraints.

\section{The Lagrangian of the model}

Suppose that the gauge symmetry of the theory is $S U(N) \times U(2)$ group, which is written specifically as follows:

$G=S U(N) \times U(2)$

where $S U(N)$ is the special unitary group of $\mathrm{N}$-dimensions, $\psi(x)$ is a $\mathrm{N}$-component vector in the fundamental representative space of $S U(N)$ group and $T_{i} \quad\left(i=1,2, \ldots . ., N^{2}-1\right)$ denotes the representative matrices of the generators of $S U(N)$ group. The latter are Hermit and traceless. They satisfy the condition: 
where $f_{i j k}$ are structure constants of the $S U(N)$ group and $K$ is a constant independent of the indices $i$ and $j$ but dependent on the representation of the group. The representative matrix of a general element of the $S U(N)$ group is expressed as:

$U=e^{-i \alpha^{i} T_{i}}$

with $\alpha^{i}$ being the real group parameters. In global gauge transformations, all $\alpha^{i}$ are independent of space-time coordinates, while in local gauge transformations $\alpha^{i}$ are functions of space-time coordinates. $U$ is a unitary $N \times N$ matrix.

In order to introduce the mass term of gauge fields without violating local gauge symmetry at energy scale close to $2 \mathrm{TeV}$, two kinds of gauge fields are required: $a_{\mu}(x)$ and $b_{\mu}(x) \cdot a_{\mu}(x)$ and $b_{\mu}(x)$ are vectors in the canonical representative space of $S U(N)$ group. They can be expressed as linear combinations of generators, as follows

$a_{\mu}(x)=a_{\mu}^{i}(x) T_{i}$

$b_{\mu}(x)=b_{\mu}^{i}(x) T_{i}$

where $a_{\mu}(x)$ and $b_{\mu}(x)$ are component fields of the gauge fields $a_{\mu}(x)$ and $b_{\mu}(x)$ respectively. Corresponding to these two kinds of gauge fields, there are two kinds of gauge covariant derivatives:

$$
\begin{aligned}
& D_{\mu}=\partial_{\mu}-i g a_{\mu} \\
& D_{b \mu}=\partial_{\mu}+i c g b_{\mu}
\end{aligned}
$$

The strengths of gauge fields $a_{\mu}(x)$ and $b_{\mu}(x)$ are defined as

$$
\begin{aligned}
& a_{\mu v}=\frac{1}{-i g}\left[D_{\mu}, D_{v}\right]=\partial_{\mu} a_{\nu}-\partial_{\nu} a_{\mu}-i g\left[a_{\mu}, a_{v}\right] \\
& b_{\mu v}=\frac{1}{i c g}\left[D_{b \mu}, D_{b v}\right]=\partial_{\mu} b_{v}-\partial_{\nu} b_{\mu}+i c g\left[b_{\mu}, b_{v}\right]
\end{aligned}
$$

respectively.

Similarly, $a_{\mu}(x)$ and $b_{\mu}(x)$ can also be expressed as linear combinations of generators:

$$
\begin{aligned}
& a_{\mu v}=a_{\mu v}^{i} T_{i} \\
& b_{\mu \nu}=b_{\mu v}^{i} T_{i}
\end{aligned}
$$

Using relations (2) and (8), (9), we obtain 
$a_{\mu \nu}^{i}=\partial_{\mu} a_{\nu}^{i}-\partial_{\nu} a_{\mu}^{i}+g f^{i j k} a_{\mu}^{j} a_{\nu}^{k}$

$b_{\mu \nu}^{i}=\partial_{\mu} b_{v}^{i}-\partial_{\nu} b_{\mu}^{i}-c g f^{i j k} b_{\mu}^{j} b_{v}^{k}$

The Lagrangian density of the model is

$$
\begin{aligned}
& \mathfrak{I}_{W u}=-\bar{\psi}\left(\gamma^{\mu} D_{\mu}+m\right) \psi-\frac{1}{4 K} \operatorname{Tr}\left(a^{\mu v} a_{\mu \nu}\right)-\frac{1}{4 K} \operatorname{Tr}\left(b^{\mu v} b_{\mu \nu}\right) \\
& -\frac{\mu^{2}}{2 K\left(1+c^{2}\right)} \operatorname{Tr}\left[\left(a^{\mu}+c b_{\mu}\right)\left(a_{\mu}+c b_{\mu}\right)\right]
\end{aligned}
$$

where $c$ is a constant.

The space-time metric is selected as $\eta_{\mu v}=\operatorname{diag}(-1,1,1,1),(\mu, v=0,1,2,3)$. According to relation (2), the above Lagrangian density $\mathrm{L}$ can be rewritten as:

$$
\begin{aligned}
& \mathfrak{I}_{W u}=-\bar{\psi}\left[\left(\gamma^{\mu} \partial_{\mu}-i g a_{\mu}^{i} T_{i}\right)+m\right] \psi-\frac{1}{4} a^{i \mu v} a_{\mu \nu}^{i}-\frac{1}{4} b^{i \mu v} b_{\mu \nu}^{i} \\
& -\frac{\mu^{2}}{2\left(1+c^{2}\right)}\left(a^{i \mu}+c b^{i \mu}\right)\left(a_{\mu}^{i}+c b_{\mu}^{i}\right)
\end{aligned}
$$

In equation (1), the gauge group $U(2)=S U_{L}(2) \times U(1)_{Y}$ is the known SM of electroweak (EW) interactions [20], [21], [22], [23]. The generators of $S U_{L}(2)$ correspond to the three components of weak isospin $T_{i}\left((i=1,2,3)\right.$ The $U(1)_{Y}$ generator corresponds to the weak hypercharge $Y$. These are related to the electric charge by $Q=\frac{T_{3}}{2}+Y$.

The $S U_{L}(2) \times U(1)_{Y}$ invariant Lagrangian is given as follows

$$
\mathfrak{\Im}_{E W}=\overline{\psi \gamma}{ }^{\mu}\left[i \partial_{\mu}-g \frac{1}{2} \tau \llbracket A_{\mu}+\frac{g^{\prime}}{2} B_{\mu}\right] \psi+\bar{e}_{R} \gamma^{\mu}\left[i \partial_{\mu}+g^{\prime} B_{\mu}\right] e_{R}-\frac{1}{4} F_{\mu \nu}^{\alpha} F_{\alpha}^{\mu \nu}-\frac{1}{4} B_{\mu \nu} B^{\mu \nu}
$$

with the field strength tensors

$$
\begin{aligned}
& F_{\mu \nu}^{\alpha}=\partial_{\mu} A_{\nu}^{a}-\partial_{\nu} A_{\mu}^{a}-g \varepsilon^{\alpha \beta \gamma} A_{\mu}^{\beta} A_{\nu}^{\alpha} \\
& B_{\mu \nu}=\partial_{\mu} B_{v}-\partial_{\nu} B_{\mu}
\end{aligned}
$$

for the three non-abelian fields of $S U(2)_{L}$ and the single Abelian gauge field associated with $U(1)_{Y}$, respectively. The covariant derivative is

$$
D_{\mu}=\partial_{\mu}+\frac{1}{2} i g A_{\mu}^{\alpha} T_{\alpha}+\frac{1}{2} i g^{\prime} B_{\mu}
$$


with $g, g^{\prime}$ being the $S U(2)_{L}$ and $U(1)_{Y}$ the coupling strength, respectively. This Lagrangian is invariant under the infinitesimal local gauge transformations for $S U(2)_{L}$ and $U(1)_{Y}$ independently. Being in the adjoint representation, the $S U(2)_{L}$ massless gauge fields form a weak isospin triplet with the charged fields being defined by

$W_{\mu}^{ \pm}=\left(A_{\mu}^{1} \pm i A_{\mu}^{2}\right) / \sqrt{2}$

The neutral component of $A_{\mu}^{3}$ mixes with the Abelian gauge field $B_{\mu}$ to form the physical states

$Z_{\mu}=A_{\mu}^{3} \cos \theta_{w}+B_{\mu} \sin \theta_{w}$

$A_{\mu}=B_{\mu} \cos \theta_{w}-A_{\mu}^{3} \sin \theta_{w}$

where $\tan \theta_{w}=\frac{g^{\prime}}{g}$ is the weak mixing angle.

Based on the gauge group $S U(N) \times U(2)$, the final Lagrangian of the model is given as follows

$$
\begin{aligned}
& \mathfrak{I}_{\text {Model }}=\overline{\psi \gamma}^{\mu}\left[i \partial_{\mu}-g \frac{1}{2} \tau \llbracket A_{\mu}+\frac{g^{\prime}}{2} B_{\mu}\right] \psi-\bar{\psi}\left[\left(\gamma^{\mu}-i g a_{\mu}^{i} T_{i}\right)+m\right] \psi+\bar{e}_{R} \gamma^{\mu}\left[i \partial_{\mu}+g^{\prime} B_{\mu}\right] e_{R} \\
& -\frac{1}{4} F_{\mu \nu}^{\alpha} F_{\alpha}^{\mu \nu}-\frac{1}{4} B_{\mu \nu} B^{\mu \nu}-\frac{1}{4} a^{i \mu \nu} a_{\mu \nu}^{i}-\frac{1}{4} b^{i \mu \nu} b_{\mu \nu}^{i} \\
& -\frac{\mu^{2}}{2\left(1+c^{2}\right)}\left(a^{i \mu}+c b^{i \mu}\right)\left(a_{\mu}^{i}+c b_{\mu}^{i}\right)
\end{aligned}
$$

where $c$ is a constant.

\section{The masses of gauge fields}

Obvious characteristics of the Wu Lagrangian equation (15) is that the mass term of the gauge fields is introduced into the Lagrangian and that this term does not affect the symmetry of the Lagrangian. It has been proved that the above Lagrangian has strict local gauge symmetry [16]. Because both vector fields $a_{\mu}$ and $b_{\mu}$ are standard gauge fields, this model is a gauge field model which describes gauge interactions between gauge fields and matter fields [16].

The mass term of gauge fields can be written as follows [16]:

$\left(a^{\mu}, b^{\mu}\right) M\left(\begin{array}{l}a^{\mu} \\ b^{\mu}\end{array}\right)$

where $\mathrm{M}$ is the mass matrix: 


$$
M=\frac{1}{1+c^{2}}\left(\begin{array}{cc}
\mu^{2} & c \mu^{2} \\
c \mu^{2} & c^{2} \mu^{2}
\end{array}\right) \quad .
$$

Physical particles generated from gauge interactions are eigenvectors of mass matrix and the corresponding masses of these particles are eigenvalues of mass matrix. The mass matrix $M$ has two eigenvalues

$$
m_{1}^{2}=\mu^{2} \quad, \quad m_{2}^{2}=0 .
$$

The corresponding eigenvectors are

$\left(\begin{array}{c}\cos \theta \\ \sin \theta\end{array}\right) \quad\left(\begin{array}{r}-\sin \theta \\ \cos \theta\end{array}\right)$

where,

$$
\cos \theta=\frac{1}{\sqrt{1+c^{2}}}, \quad \sin \theta=\frac{c}{\sqrt{1+c^{2}}}
$$

We define

$C_{\mu}=\cos \theta a_{\mu}+\sin \theta b_{\mu}$,

$F_{\mu}=-\sin \theta a_{\mu}+\cos \theta b_{\mu}$

$C_{\mu}$ and $F_{\mu}$ are eigenstates of mass matrix: they describe the particles generated from gauge interactions. The inverse transformations of (29) are

$$
\begin{aligned}
& a_{\mu}=\cos \theta C_{\mu}-\sin \theta F_{\mu}, \\
& b_{\mu}=\sin \theta C_{\mu}+\cos \theta F_{\mu}
\end{aligned}
$$

Taking equations (29) and (30) into account, the Wu Lagrangian density $\mathrm{L}$ given by (15) changes into:

$$
\mathfrak{J}_{W u}=\mathfrak{J}_{W u}^{(0)}+\mathfrak{I}_{W u}^{(I)}
$$

where

$$
\mathfrak{J}_{W u}^{(0)}=-\bar{\psi}\left(\gamma^{\mu} \partial_{\mu}+m\right) \psi-\frac{1}{4} C_{0}^{i \mu v} C_{0 \mu v}^{i}-\frac{1}{4} K_{0}^{i \mu v} K_{0 \mu v}^{i}-\frac{\mu^{2}}{2} C^{i \mu} C_{\mu}^{i},
$$




$$
\begin{aligned}
& \mathfrak{J}_{W u}^{(I)}=i g \overline{\psi \gamma}{ }^{\mu}\left(\cos \theta C_{\mu}-\sin \theta F \mu\right) \psi-\frac{\cos 2 \theta}{2 \cos \theta} g{ }^{i j k} C_{0}^{i \mu v} C_{\mu}^{i} C_{v}^{k}+\frac{\sin \theta}{2} g f^{i j k} K_{0}^{i \mu v} K_{v}^{i} K_{v}^{k} \\
& -\frac{\sin \theta}{2} g f^{i j k} K_{0}^{i \mu \nu} C_{\mu}^{i} C_{v}^{k}+g \sin \theta f^{i j k} C_{0}^{i \mu v} C_{\mu}^{j} K_{v}^{k}-\frac{1-\frac{3}{4} \sin ^{2} 2 \theta}{4 \cos ^{2} \theta} g^{2} f^{i j k} f^{i l m} C_{\mu}^{j} C_{\nu}^{k} C^{l \mu} C^{m v} \\
& -\frac{\sin ^{2} \theta}{2} g^{2} f^{i j k} f^{i l m} K_{\mu}^{j} K_{v}^{k} K^{l m} K^{m v}+g^{2} t c \theta \sin \theta f^{i j k} f^{i l m} C_{\mu}^{j} C_{\nu}^{k} C^{l m} K^{m v} \\
& -\frac{\sin ^{2} \theta}{2} g^{2} f^{i j k} f^{i l m}\left(C_{\mu}^{j} C_{v}^{k} K^{l \mu} K^{m v}+C_{\mu}^{j} K_{v}^{k} K^{\mu} C^{v m}+C_{\mu}^{j} K_{v}^{k} C^{l \mu} K^{m v}\right)
\end{aligned}
$$

In the above relations, we have used the following simplified notations:

$$
\begin{aligned}
& C_{0 \mu \nu}^{i}=\partial_{\mu} C_{\nu}^{i}-\partial_{\nu} C_{\mu}^{i} \\
& K_{0 \mu \nu}^{i}=\partial_{\mu} F_{\nu}^{i}-\partial_{\nu} F_{\mu}^{i}
\end{aligned}
$$

From equation (32) it is deduced that the mass of field $C_{\mu}$ is $\mu$ and the mass of gauge field $F_{\mu}$ is zero. That is

$$
M_{C}=\mu=g_{C} v \quad, \quad M_{F}=0
$$

where $g_{C}$ is the coupling constant of the $C$-boson and $v$ the vacuum expectation value. Transformations (29) and (30) are pure algebraic operations which do not affect the gauge symmetry of the Lagrangian [16]. They can, therefore, be regarded as redefinitions of gauge fields. The local gauge symmetry of the Lagrangian is still strictly preserved after field transformations. In other words, the symmetry of the Lagrangian before transformations is completely the same as the symmetry of the Lagrangian after transformations. We do not introduce any kind of symmetry breaking at energy scales close to $2 \mathrm{TeV}$.

Fields $C_{\mu}$ and $F_{\mu}$ are linear combinations of gauge fields $a_{\mu}$ and $b_{\mu}$, so the forms of local gauge transformations of fields $C_{\mu}$ and $F_{\mu}$ are determined by the forms of local gauge transformations of gauge fields $a_{\mu}$ and $b_{\mu}$. Because $C_{\mu}$ and $F_{\mu}$ consist of gauge fields $a_{\mu}$ and $b_{\mu}$ and transmit gauge interactions between matter fields, for the sake of simplicity we also call them gauge fields, just as $\mathrm{W}$ and $\mathrm{Z}$ are called gauge fields in the electroweak model ([20], [21], [22], [23]). This gauge field theory, therefore, predicts the existence of two different kinds of force transmitting vector fields exist in this gauge field theory: one is massive and another is massless.

Taking the Higgs mechanism [20-23] into account, in the vacuum energy scale of $179 \mathrm{GeV}$ the $W^{ \pm}$and $Z^{0}$ become massive, while the photon $A$ remains massless. The symmetry $S U(N)$ does not break down, since the gauge bosons $C^{0}$ and $F$ get their masses by the $\mathrm{Wu}$ mechanisms [16], [17], [18], [19] in the vacuum energy scale of $2 \mathrm{TeV}$.

The EW Lagrangian (16) changes into:

$$
\begin{aligned}
& \mathfrak{J}_{E W}=\left(D_{\mu} \Phi\right)^{+}\left(D_{\mu} \Phi\right)-\mu^{2} \Phi^{+} \Phi-\lambda\left(\Phi^{+} \Phi\right)^{2} \\
& -\frac{1}{4} F_{\alpha}^{\mu v} F_{\mu v}^{\alpha}-\frac{1}{4} B^{\mu v} B_{\mu v}
\end{aligned}
$$


The covariant derivatives are given by the equation (19) which acts on a complex $S U$ (2) field:

$$
\Phi=\frac{1}{\sqrt{2}}\left(\begin{array}{l}
\phi_{1}+i \phi_{1} \\
\phi_{3}+i \phi_{4}
\end{array}\right)
$$

Let us now introduce the vacuum expectation value of $179 \mathrm{GeV}$, which violates local gauge symmetry of the Lagrangian.

$$
<\Phi>=\frac{1}{\sqrt{2}}\left(\begin{array}{l}
0 \\
v
\end{array}\right),
$$

$$
\begin{aligned}
& <\Phi>=\frac{1}{\sqrt{2}} U^{-1}(\varsigma)\left(\begin{array}{c}
0 \\
v+\eta
\end{array}\right) \\
& U(\varsigma)=\exp \left(\frac{-i \varsigma \tau}{2 v}\right) \\
& \Phi \rightarrow \Phi^{\prime}=U(\varsigma) \Phi=\frac{1}{\sqrt{2}}\left(\begin{array}{c}
0 \\
v+\eta
\end{array}\right)
\end{aligned}
$$

From the above we calculate:

$$
\begin{aligned}
& \mathfrak{J}_{E W}=\left(\partial_{\mu} \eta\right)\left(\partial^{\mu} \eta\right)-\frac{1}{2} \mu^{2}(v+\eta)^{2}-\frac{1}{4} \lambda(v+\eta)^{2}-\frac{1}{4} F_{\alpha}^{\mu v} F_{\mu v}^{\alpha}-\frac{1}{4} B^{\mu v} B_{\mu v} \\
& +\frac{1}{4} \Phi^{+}\left(g^{\prime} B_{\mu}+g \tau A_{\mu}\right)\left(g^{\prime} B^{\mu}+g \tau A^{\mu}\right) \Phi^{\prime}
\end{aligned}
$$

The EW Lagrangian $\mathfrak{I}_{E W}$ can be written as the sum of classical part, a part quadratic in the fields and a part cubic and quadratic in the fields, which gives rise to interactions.

By the definitions of the gauge fields equations (20), (21), (22), the masses are given as follows

$$
M_{A}=0, M_{W}=\frac{1}{2} g v, M_{Z}=\frac{1}{2} \sqrt{g^{2}+g^{\prime 2}}
$$

The gauge fields and masses predicted by this model are summarized in Table.1.

Table.1. Gauge fields and masses predicted by the present model.

\begin{tabular}{|l|l|l|l|}
\hline Gauge fields & Masses (M)-GeV & Vacuum (v)-GeV & Symmetry patter \\
\hline C (New) & 145 & 2000 & Before -symmetry breaking \\
\hline F (New) & 0 & 2000 & Before- symmetry breaking \\
\hline W & 81 & 179 & After -symmetry breaking \\
\hline Z & 91 & 179 & After - symmetry breaking \\
\hline A & 0 & 179 & After- symmetry breaking \\
\hline
\end{tabular}




\section{The Phenomenology of the model}

The phenomenology of the proposed $\mathrm{C}$ gauge boson is similar to the Z-prime, a hypothetical carrier of a new force similar to the electroweak force [29].

Following ref [29], the coupling constant $g$ is the SM coupling $g / \cos \theta_{w}$. For our setup, $g_{C}$ is related to $g$ by

$$
\frac{g_{C}}{g}=\left(\frac{5}{3} x_{w} \lambda\right)^{1 / 2} \square 0.63 \lambda^{1 / 2},
$$

where $x_{w}=\sin ^{2} \theta_{w}$ and $\theta_{w}$ is the weak mixing angle. The decay width of $C$ gauge boson into fermions is given by

$$
\Gamma(C \rightarrow \overline{f f})=\frac{G_{F} M_{C}^{2}}{6 \pi \sqrt{2}} N_{c} C\left(M_{C}^{2}\right) M_{C} \sqrt{1-4 x}\left[u_{n}^{f_{2}}(1+2 x)+a_{n}^{f_{2}}(1-4 x)\right]
$$

where $G_{F}$ is the Fermi coupling constant, and $N_{c}=3$ or 1 , if $f$ is a quark or a lepton, respectively.

The term $\mathrm{C}$ is given by the following equation:

$$
\begin{aligned}
& C\left(M_{C}^{2}\right)=1 / \alpha_{s} / \pi+1.409\left(\alpha_{s} / \pi\right)^{2}-12.77\left(\alpha_{s} / \pi\right)^{3}, \\
& \alpha_{s}=\alpha_{s}\left(M_{C}\right)
\end{aligned}
$$

C is the strong coupling at the scale $M_{C}, x=m_{f}^{2} / M_{C}^{2}$, The $C$ width is proportional to $\lambda$, which sets the strength of the $C$ coupling. For $\lambda=1$ the total $C$ width is

$$
\Gamma_{C} / M_{C}=0.022 \quad \text { for } \quad M_{C}<2 m_{t}
$$

This width will be increased somewhat if there are open channels for decay into the top quark, superpartners, and other exotic particles.

The $\mathrm{C}$ boson can be directly produced at a hadron collider via the $\bar{q} q \rightarrow C$ subprocess, for which the cross section in the narrow $\mathrm{C}$ width approximation is

$$
\hat{\sigma}(q \bar{q} \rightarrow C)=K \frac{2 \pi}{3} \frac{G_{F} M_{C}^{2}}{\sqrt{2}}\left[\left(u_{n}^{q}\right)^{2}+\left(a_{n}^{q}\right)^{2}\right] \delta\left(\hat{s}-M_{C}^{2}\right)([24])
$$

The K-factor represents the enhancement from higher-order QCD processes, estimated to be

$$
K=1+\frac{\alpha_{s}\left(M_{C}^{2}\right)}{2 \pi} \frac{4}{3}\left(1+\frac{4}{3} \pi^{2}\right) \square 1.3([24])
$$

When mixing is ignored it is,

$$
\left(u_{n}^{q}\right)^{2}+\left(a_{n}^{q}\right)^{2}=(0.62)^{2} \lambda
$$


and the above cross section is independent of the parameter $\gamma$ as long as $\varepsilon_{V}^{2}+\varepsilon_{A}^{2}=1$.

Note that all the current and previous dijet-mass searches at the Tevatron are limited to $M_{j j}>200 \mathrm{GeV}$. These energy levels are not applicable to the present $C$ with $M_{C} \approx 145 \mathrm{GeV}$.

The relevant dijet data are from the UA2 collaboration, with collision energy at $\sqrt{\mathrm{s}}=630 \mathrm{GeV}$ The UA2 Collaboration [25] has detected the $W+Z$ signal in the dijet mass rage $48 \mathrm{GeV}<m(j j)<138 \mathrm{GeV}$ and has placed the upper bound of $\sigma B(C \rightarrow j j)$ over the range $80 \mathrm{GeV}<m(j j)<320 \mathrm{GeV}$.

The associated production of $C$ with a $W$ - boson goes through the $\mathrm{t}$ - and u-channel exchange of quarks, while the s-channel boson exchange is highly suppressed because of the negligible mixing angle between the SM $Z$-boson and the $C$. Consequently, we expect similar or even larger cross sections for $M_{C} \square M_{Z}$ than the SM WZ production in which there is a delicate gauge cancellation among the t-, $\mathrm{u}$-, and s-channel diagrams. We have included a $K$-factor ( $K=1.3$ to approximate next-to-leading order QCD contributions [26]. We can see that, at around $140-150 \mathrm{GeV}$, the cross section is right at the order of $4 \mathrm{pb}$, which is required to explain the excess in the CDF $W j j$ anomaly [2].

\section{Conclusion}

We have shown that a new neutral vector $C$ - boson of mass $(145 \mathrm{GeV})$, predicted by the $\mathrm{Wu}$ mechanisms for mass generation of gauge field, can explain the excess in the invariant-mass window $120-160 \mathrm{GeV}$ in the dijet system of $\mathrm{Wjj}$ production.

In this model, the Standard Model (SM) W,Z-bosons acquire their masses through the coupling with the SM Higgs of mass $114-200 \mathrm{GeV}$. The $C$-boson has negligible couplings to leptons, and so is not affected by the dilepton $C$ constraints.

\section{References}

[1]. T. Aaltonen et al. [CDF Collaboration] 2008 Preprint hep-ex/ $\underline{0806.2472}$

[2]. T. Aaltonen et al. [CDF Collaboration] 2011 Preprint hep-ex/1101.0034

[3]. V. M. Abazov et al. [DØ Collaboration] 2008 Preprint hep-ex/0712.0851

[4]. T. Aaltonen et al. [CDF Collaboration] 2011 Preprint hep-ex / $\underline{1104.0699}$

[5]. B. Holdom 1981 Phys. Rev. D24 , 1441

[6]. T. W. Appelquist, D. Karabali, and L. C. R. Wijewardhana 1986 Phys. Rev. Lett. 57,957

[7]. K. Yamawaki, M. Bando, and K.-i. Matumoto 1986 Phys. Rev. Lett. 56 , 1335

[8]. T. Akiba and T. Yanagida 1986 Phys. Lett. B169 , 432

[9]. J. Goldstone 1961 Nov. Cim. 19, 154

[10]. Y Nambu, G.Jona-Lasinio 1961 Phys.Rev. 122,34

[11]. J.Goldstone, A.Salam, S.Weinberg. 1970 Phys.Rev.127 
[12]. P.W.Higgs 1964 Phys.Lett.12, 13218

[13]. F.Englert, R.Brout 1964 Phys.Rev.Lett. 13, 321

[14]. G.S.Guralnik, C.R.Hagen, T.W.B.Kibble 1964 Phys.Rev.Lett 585

[15]. P.W.Higgs 1966 Phys.Rev. 145, 1156

[16]. Ning Wu 2000 Preprint hep-ph/ㅁ005072

[17]. Ning Wu 1998 Preprint hep-ph/ㅆ802236

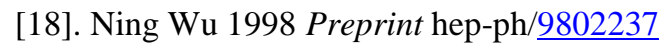

[19]. Ning Wu 1998 Preprint hep-ph/ㅆ805453

[20]. S. Weinberg 1967 Phys. Rev. Lett. 19, 1264

[21]. S. Weinberg 1972 Phys. Rev. D 5, 1412

[22]. A. Salam 1968 In Elementary Particle Theory: Relativistic Groups and Analyticity (Nobel Symposium No. 8, ed. N. Svartholm (Almqvist and Wiksell, Stockholm)

[23]. S. Glashow 1961 Nucl. Phys. 22, 579

[24]. V. Barger and R.J.N. Phillips 1987 Collider Physics (Addison-Wesley)

[25]. J. Alitti et al. [UA2 Collaboration] 1991 Z. Phys. C 49, 17

[26] J. Ohnemus 1993 Phys. Rev. D47, 940

[27].V.M. Abazov et al. [D0 Collaboration] 2011 Preprint hep-ex / $\underline{1106.1921}$

[28]. Graham G. Ross 2001 Structure beyond the Standard Model Phil. Trans. R. Soc. Lond. A 359, 405-419

[29]. Kingman.C, Jeonghyeon.S 2011 Preprint hep-ph/1104.1375 